\title{
Dynamic Programming Strategies on the Decision Tree Hidden behind the Optimizing Problems
}

\author{
Zoltan KATAI \\ Mathematics and Informatics Department, Sapientia Hungarian University of Transylvania \\ Bd. 1848, 58/30, 540398, Targu Mures, Romania \\ e-mail: katai_zoltan@ms.sapientia.ro
}

Received: April 2006

\begin{abstract}
The aim of the paper is to present the characteristics of certain dynamic programming strategies on the decision tree hidden behind the optimizing problems and thus to offer such a clear tool for their study and classification which can help in the comprehension of the essence of this programming technique.
\end{abstract}

Key words: dynamic programming, programming techniques, decision trees.

\section{Introduction}

Several books treat the problem of dynamic programming by presenting the principles standing at the basis of the technique and then giving a few examples of solved problems. For instance the book called Algorithms by Cormen, Leiserson and Rivest (1990), mentions the optimal substructures and the overlapped subproblems as elements of the dynamic programming. Răzvan Andone and Ilie Garbacea (1995) are talking about the three basic principles of the dynamic programming in their book Basic Algorithms:

1) avoid the repeated solving of identical subproblems by saving the optimal subsolutions;

2) we solve the subproblems advancing from the simple toward the complex;

3) the principle of optimality.

The book Programming Techniques by Tudor Sorin (1997) gives a certain classification of the dynamic programming strategies: forwards method, backwards method and mixed method.

In this paper we would like to go further in the study and classification of the dynamic programming strategies. By presenting the characteristics of certain dynamic programming strategies on the decision tree hidden behind the optimizing problems we offer such a clear tool for their study and classification which can help in the comprehension of the essence of this programming technique. 


\section{The Decision Tree}

Dynamic programming is often used to solve optimizing problems. Usually the problem consists on a target function which has to be optimized through a sequence of (optimal) decisions. For each optimizing problem a rooted tree (tree structure) can be ordered, which will be called decision tree. The root represents the starting state of the problem, the first level nodes represent the states the problem can reach after the first decision, the second level nodes those reached after the second decision etc. A node will have as many sons as the number of possible choices for the respective decision.

Fig. 1 presents a situation when the solution was obtained after four decisions. At each decision there was a choice of two possibilities. The labels of the nodes identify the corresponding states.

We dissociate two cases:

I type decision tree: By each decision the problem is reduced to a similar problem of smaller size, represented by one of the subtrees of the current node. In this situation the optimal solution will be represented by one of the root-leaf paths on the decision tree. The above picture is referring to such a situation. The dotted rectangles show the way in which - in case of the bold arrows road is the optimal - the problem is reduced to its smaller and smaller subproblems.

II type decision tree: By each decision the problem is divided into two or more smaller sized subproblems, represented by the corresponding subtrees of the current node. Fig. 2 shows such a situation when by each decision the problem is broken down into two subproblems. We supposed that the bold binary subtree represents the breakdown into optimal subproblems. We will call the version of the dynamic programming referring to this situation "Optimal division - optimal conquest". Why is this name suggestive we will clarify later.

Making a decision means to choose. According to the choice at each decision we get different subproblems. We can thus say that the subtrees of a decision tree represent those

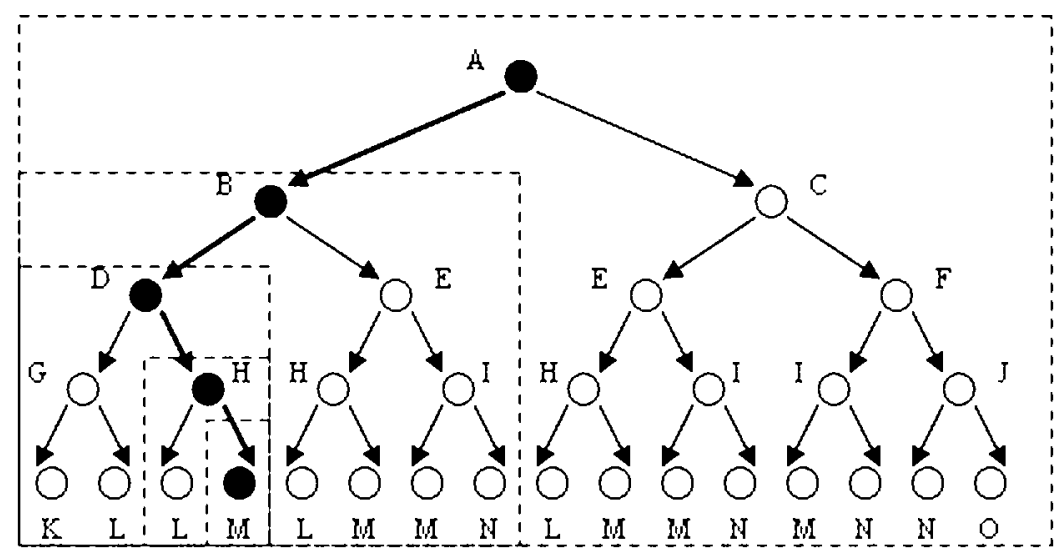

Fig. 1. I type decision tree. 


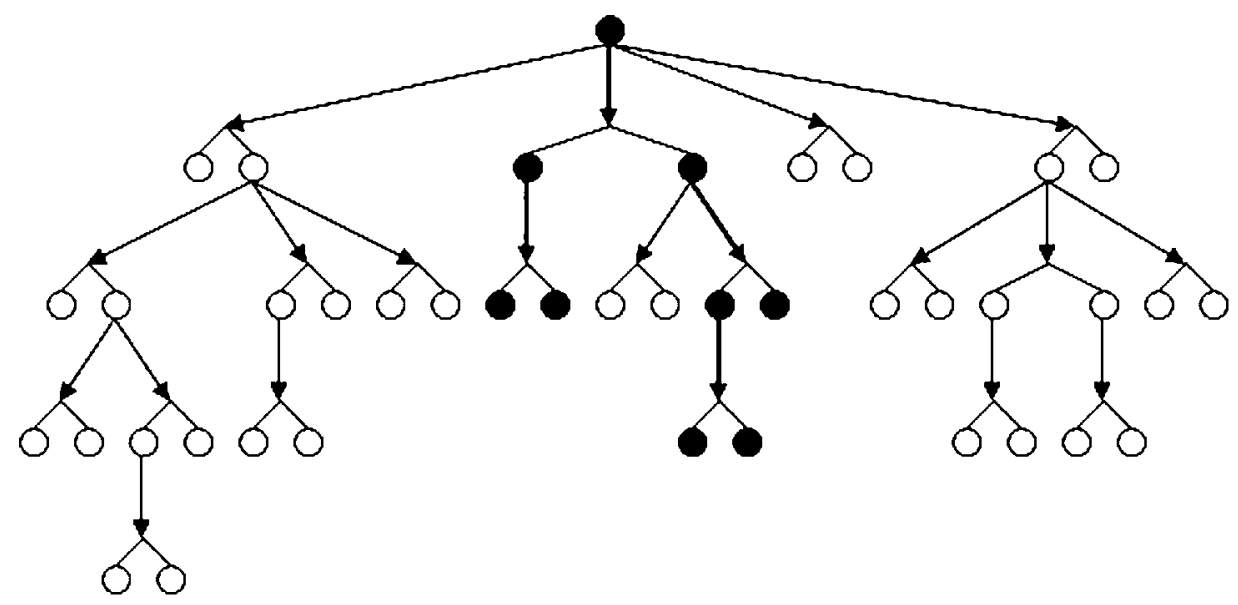

Fig. 2. II type decision tree.

subproblems the problem can be reduced to (case 1), respectively broken down (case 2) through certain sequences of decisions.

Considering the fact that the subproblems are similar, we can speak about their general form. By general form we always mean a form with parameters. To comprehend the structure of a problem means, amongst others, to clarify the followings:

- what is the general form of the subproblems,

- which parameters describe this,

- which are the parameter values for which we get the original problem, respectively the trivial subproblems as marginal cases of the general problem.

\section{The Contracted Decision Tree}

Although the number of the decision tree's nodes depends exponentially on the number of the decisions of the optimal sequence of decisions, it often happens that it contains several identical nodes, which obviously represent identical states and which are of course characterized by the same parameter values. (As we will later see, the bigger the number of identical nodes, the more can the dynamic programming make use of its strengths.) In case of the first type decision trees this situation occurs when the problem is reduced to the same subproblem by means of different subsequences of decisions. Such a situation is represented by the decision tree shown in Fig. 1. We overlap the nodes representing the identical states of the tree (see Fig. 3). We called the obtained data structure contracted decision tree.

As we can see, the contracted decision tree is not a tree structure any more, but a digraph. The contracted tree will have exactly as many nodes as the number of different states the problem can get (this number is usually only a polynomial function of the number of decisions leading to the optimal solution.). Although we have presented the 


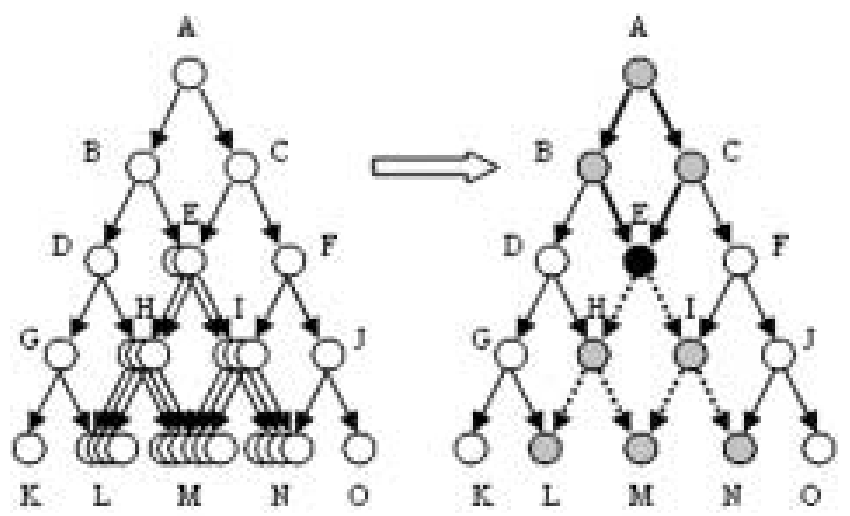

Fig. 3. Contracted decision tree.

notion of the contracted decision tree connected to the first case, we will see that it can be extended to the second type decision trees as well.

\section{The Theory of Optimality}

The dynamic programming is built on the principle of optimality. We could even say that it is the implementation of this basic principle. We can express the principle of optimality in the following way: the optimal solution is built by optimal subsolutions. In other words the optimal solution of the problem can be built with the optimal solutions of the subproblems. The dynamic programming follows this strategy: starts from the optimal solutions of the trivial subproblems and builds the optimal solutions of the more and more complex subproblems and eventually of the original problem. This is why we say that it advances from the simple to the complex or that it solves the problem from the bottom to the top (bottom-up way).

An other feature of the dynamic programming is that it records the optimal values of the optimal solutions of the already solved subproblems (the optimal values of the target function to be optimized concerning the respective subproblems). For this we are generally using an array we are going to represent with $c$. This array will have one, two or more dimensions depending on the number of parameters describing the general form of the subproblems. The number of the used elements of this array is of course identical to the number of the nodes of the contracted decision tree, namely to the number of different subproblems.

The basic principle of optimality is expressed by a recursive formula, which describes mathematically the way in which the solutions of the more and more complex subproblems are built from the simpler subproblems' optimal solutions. Obviously this is a formula where the way of the optimal decision making has been built in. The recursive formula has been drafted for the elements of the array $c$, so it is working with the optimal values of the subproblems. 
How is the implementation of the basic principle of optimality reflected on the decision tree, on the contracted decision tree, respectively on the array storing the optimal values of the subproblems?

1. If nodes representing identical states appear on the crown of the growing decision tree, it will grow only in the direction of the branch representing the optimal solution of the respective subproblem.

2. We prune the nodes of the contracted decision tree, in the order dictated by the recursive formula, in such a manner that there should be only one path to each of them, namely the one representing the optimal solution.

3. The core of the algorithm is represented by the filling of the corresponding elements of the $c$ array according to the strategy given by the recursive formula. Although the $c$ array stores one to one only the optimal values of the subproblems, still it contains enough information to reconstruct the sequence of the optimal decisions. It is often suitable to somehow store the optimal choices themselves when filling the array $c$. This could facilitate or even make faster the reconstruction of the optimal decision-sequence.

When the principle of optimality is valid for a problem, this could considerably reduce the time necessary to build the optimal solution, because in building it we can rely exclusively on the optimal solutions of the subproblems.

\section{Dynamic Programming on the I Type Decision Tree}

Let's picture to ourselves again the first type decision tree. The root represents the starting state, when the whole problem needs to be solved. The solution-leaves of the tree represent the solved states of the problem and the root-solutionleaf paths represent the potential solutions (the wanted optimal solution is to be found among them). In the intermediate states represented by the nodes of the tree the problem contains a part which has already been solved and one which needs to be solved. We call these the prefix and suffix subproblems of the respective state.

The path leading to a given node represents the sequence of decisions leading to the corresponding state. This sequence of decisions can be considered as one solution of the prefix subproblem of the respective state. The different sequences of decisions leading to the identical nodes overlapped in the contracted decision tree can be considered as different solutions of the corresponding prefix subproblem.

The suffix subproblem of a state (this is nothing else but the subproblem the problem in the respective state has been reduced to) - as we have already referred to it - is represented in the decision tree by the subtree of the corresponding node (that one whose root is the node). The solutions of the suffix type subproblems are obviously represented by the root-leaf paths of the corresponding subtree. On picture number 3 the solutions of the prefix problem of state are bold, the edges of the subtree representing the corresponding suffix problem are dotted. 
The optimal solutions of the prefix respectively suffix problems belonging to the same state in the contracted tree will be represented by the optimal root-node path connected to the respective node, respectively the optimal node-leaf path.

We can distinguish two subcases, should the contracted decision tree, as a directed graph, contents or not cycles.

\section{If the Contracted Decision Tree is Cycle Free}

What does it mean to advance from simple towards the complex (from bottom to top)? From the point of view of the prefix subproblems, from bottom to top means the rootleaves direction. Considering the suffix problems, they are growing in the opposite direction, from the leaves towards the root. This duality leads to the two versions of the dynamic programming:

1. Root-leaves directed dynamic programming (Method Forwards).

2. Leaves-root directed dynamic programming (Method Backwards).

Should $D_{1}, D_{2}, \ldots, D_{i-1}, D_{i}, D_{i+1}, \ldots, D_{n}$ be the optimal sequence of decisions (in the decision tree this is leading along the root-leaf path representing the optimal solution). Let us suppose that this sequence of decisions, starting from the original state $S_{0}$ (represented by the root of the tree), "goes through" the states $S_{1}, S_{2}, \ldots, S_{i}, \ldots, S_{n}$ (state $S_{n}$ is represented by the tree's "optimal leaf"). The above mentioned two versions of the dynamic programming uses the basic principle of the optimality in the following forms:

Assuming that $D_{1}, D_{2}, \ldots, D_{n}$ is the optimal sequence of decisions meaning the solution of the problem, then

1) subsequences of decisions bearing the form $D_{1}, D_{2}, \ldots, D_{i}(i=1, n-1)$ are also optimal,

2) subsequences of decisions bearing the form $D_{i}, D_{i+1}, \ldots, D_{n}(i=2, n)$ are also optimal.

In order to have a better view of these strategies, we apply them for the following problem.

Triangle: On the main diagonal and in the triangle under the main diagonal of a square matrix with n rows there are natural numbers. We assume that the matrix is stored in a bidimensional array a. Determine the "the longest" path from peak (element a [1][1]) to the base ( $n$-th row), considering the following:

- on a certain path element $a[i][j]$ can be followed either by element $a[i+1][j]$ (down), or by element $a[i+1][j+1]$ (diagonally to the right), where $1 \leqslant i<n$ and $1 \leqslant j<n$;

- by the "length" of a path we mean the sum of the elements to be found along the path.

For example, should for $n=5$ the matrix be the following (see Fig. 4), then the "longest" path from the peak to the base is the shaded one, its length is 37.

The following decision tree can be associated to the problem (see Fig. 5). 


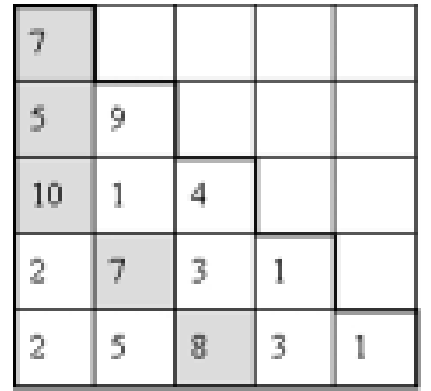

Fig. 4. Array $a$ associated to the triangle problem.

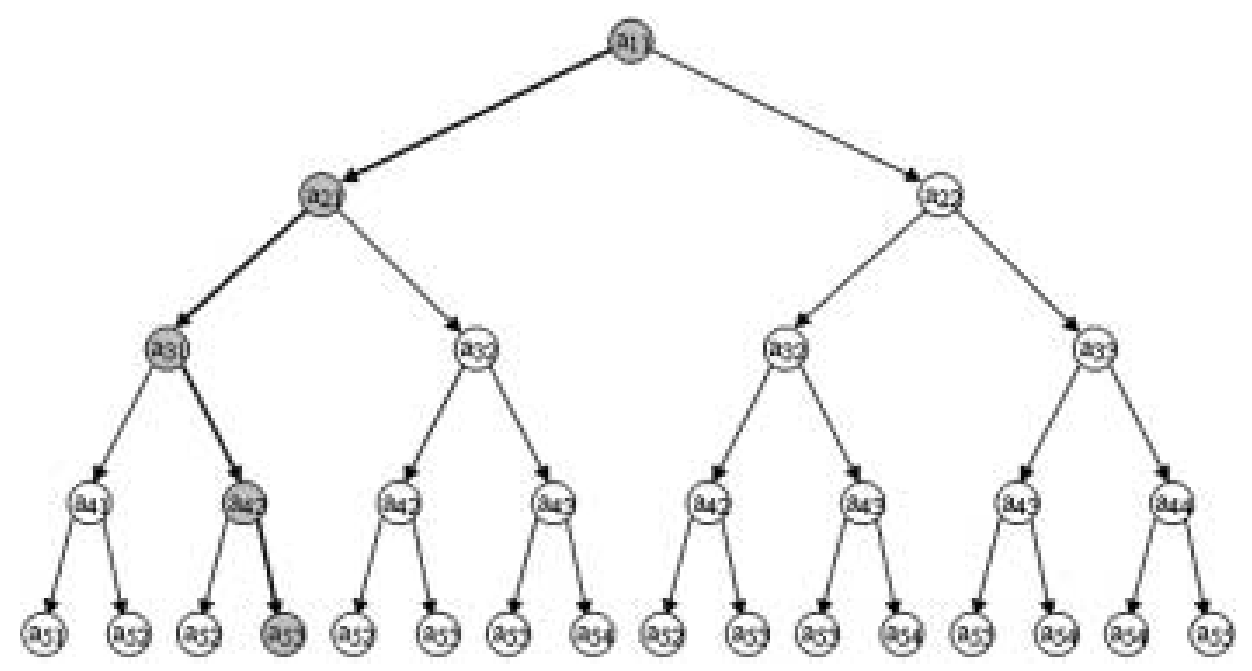

Fig. 5. The decision tree associated to the triangle problem.

The general prefix problem:

Determine the optimal path from the peak to position $(i, j)$.

The general suffix problem:

Determine the optimal path from position $(i, j)$ to the base.

So the parameters of the problem are $i$ and $j$. We can see that the decision tree has nodes with the same corresponding parameter values. Obviously these nodes represent identical states, with the same associated prefix respectively suffix problem. We overlap the nodes representing identical states. We get the attached contracted decision tree. We see it also built into the array (see Figs. 6 and 7).

As the subproblems are determined by two independent parameters, we use a bidimensional array (more exactly, the elements of the array situated on the main diagonal and under it) to store the optimal values belonging to their optimal solutions. It can be noticed that the number of the nodes of the contracted decision tree is the same as the number of the used elements of the array chosen for the storage. 


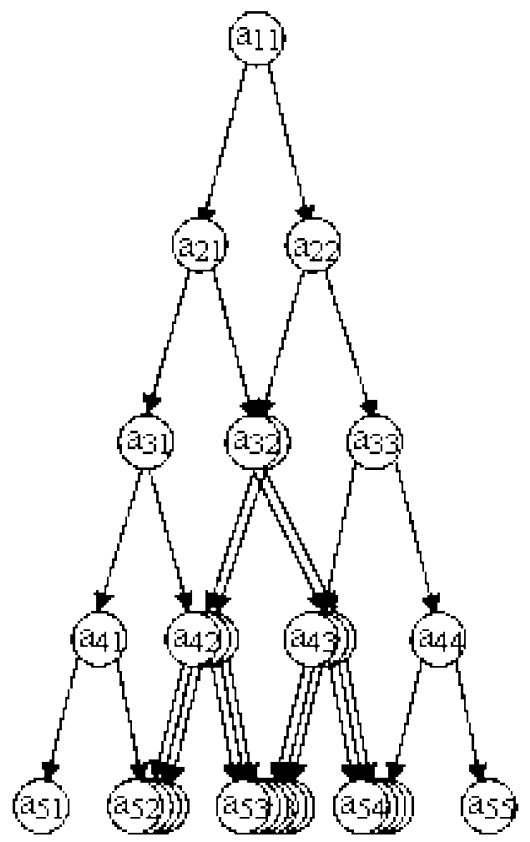

Fig. 6. The contracted decision tree attached to the triangle problem.

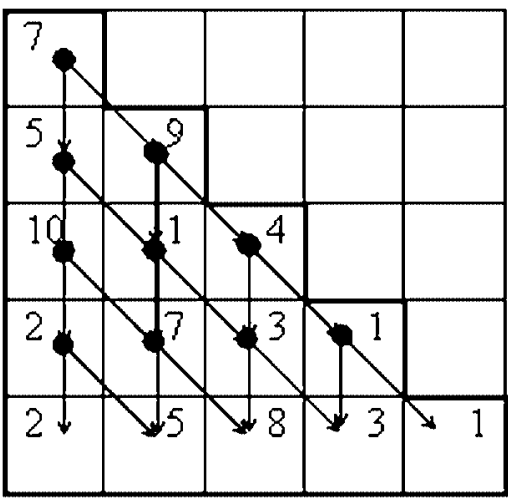

Fig. 7. The contracted decision tree built into the array.

In the root-leaves oriented version the element $c[i][j]$ of the array stores the optimal value of the prefix problem associated to state $(i, j)$, namely the length of the optimal path leading from the peak to the element with position $(i, j)$. Opposed to that, in the leaves-root oriented version the length of the best path leading from position $(i, j)$ to the base gets into the element $c[i][j]$ of the array, which represents the optimal value of the suffix problem. 


\section{Root-leaves Oriented Dynamic Programming}

We prune every node advancing from the root towards the leaves, leaving only "the best" father-branch, the one traversed by the optimal solution of the prefix problem of the respective node. What has been marked on the Fig. 8 is only one of the possible orders of pruning. The basic requirement is that when a node is the next one in order, its fathernodes should have already been pruned before. We could say that we tackle the nodes in a topological order. Since the contracted decision tree, as a digraph, is cycle free, this order exists.

The implementation of the above presented algorithm means to fill up the elements of array $c-$ in topological order, according to the following recursive formula (see Figs. 9 and 10):

$$
\begin{aligned}
& c[1][1]=a[1][1], \\
& c[i][1]=a[i][1]+c[i-1][1], \quad 2 \leqslant i \leqslant n, \\
& c[i][j]=a[i][j]+\max (c[i-1][j], c[i-1][j-1]), \quad 2 \leqslant i \leqslant n, \quad 2 \leqslant j \leqslant i .
\end{aligned}
$$

The elements of row $n$ of array $c$ store the length of the best paths from the peak to the respective positions. Obviously the biggest one represents the length of the best path from the peak to the base. If we would like to get the optimal path itself, array $c$ contains

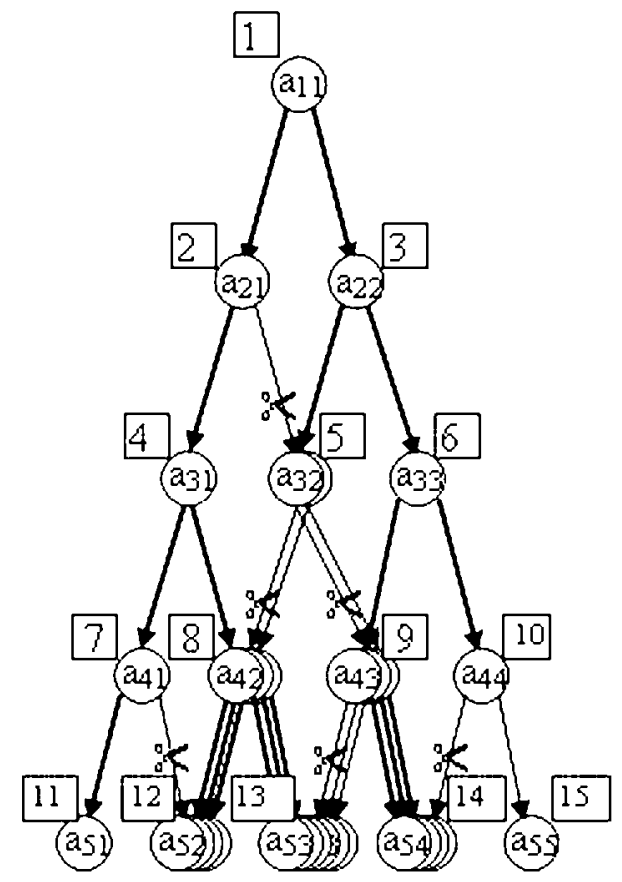

Fig. 8. A possible prune order of the contracted decision tree in case of the root-leaves oriented dynamic programming. 


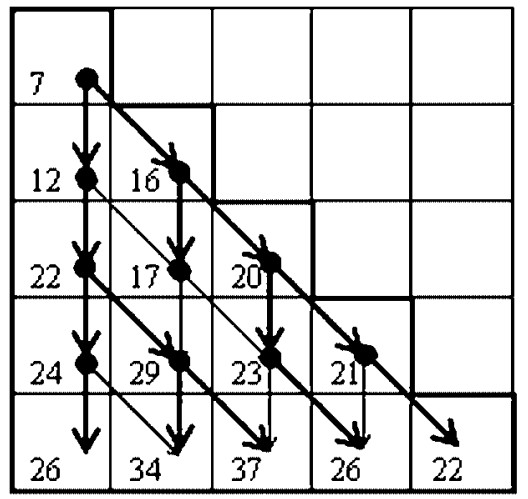

Fig. 9. The filled up array $c$ in case of the root-leaves oriented dynamic programming.
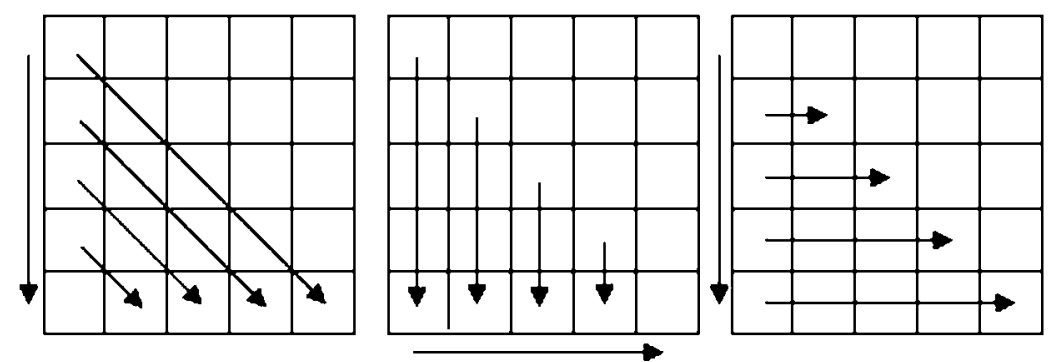

Fig. 10. Possible topological orders.

enough information in order to go back on the optimal leaf-root path. If we "climb" on the optimal path with a recursive procedure and we write out the stations on the way back of the recursive procedure, then we get the optimal solution going from the peak to the base, so to say forwards.

\section{Leaves-Root Oriented Dynamic Programming}

Advancing from the leaves towards the root we prune every node, leaving only "the best" son on it, the one which is the root of the highest optimal value son-subtree of the subtree representing the suffix problem of the respective node. Here also, what has been marked on the picture (see Fig. 11) is only one of the possible orders of pruning. In this case, the basic requirement is that when a node is the next one in order, its son-nodes should have already been pruned before.

The implementation of this algorithm can be achieved by filling up the elements of array $c$ - in an inversed topological order - according to the following recursive formula (see Fig. 12):

$$
\begin{aligned}
& c[n][j]=a[n][j] \quad 1 \leqslant j \leqslant n \\
& c[i][j]=a[i][j]+\max (c[i+1][j], c[i+1][j+1]), \quad n>i \geqslant 1, \quad 1 \leqslant j \leqslant i .
\end{aligned}
$$




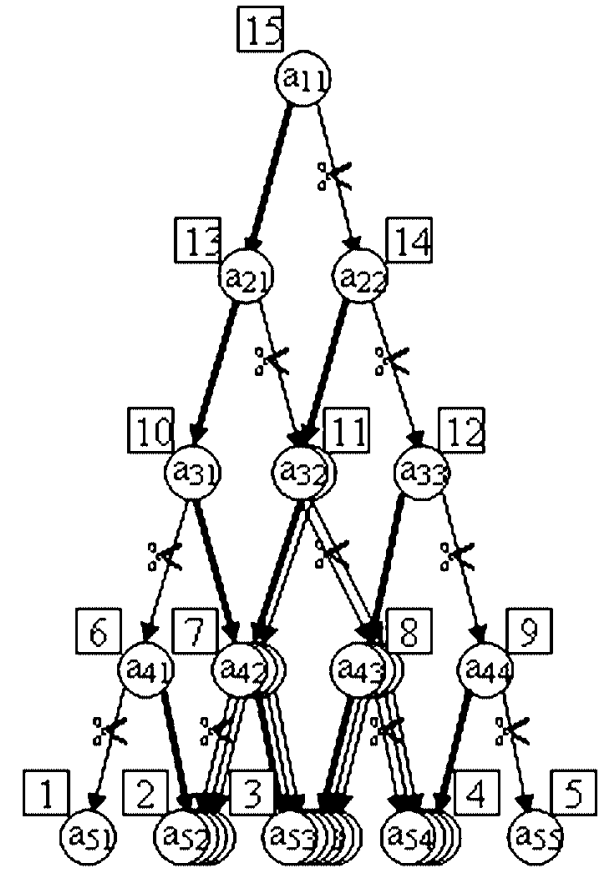

Fig. 11. A possible prune order of the contracted decision tree in case of the leaves-root oriented dynamic programming.

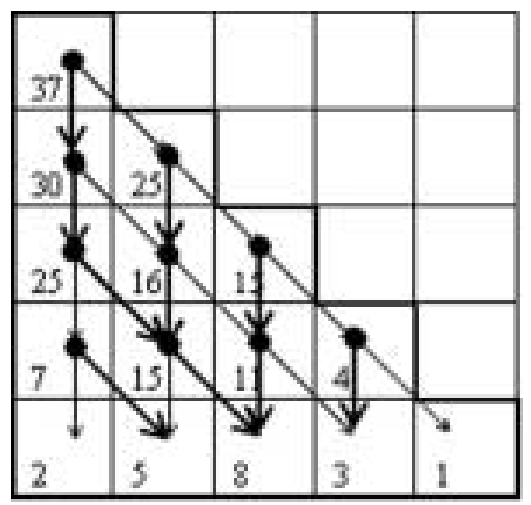

Fig. 12. The filled up array $c$ in case of the leaves-root oriented dynamic programming.

Eventually element $c[1][1]$ will store the optimal value of the original problem, as the biggest suffix problem. As array $c$ contains the optimal value of all the suffix subproblems, now we possess enough information to "run down" with a greedy algorithm on the path from the peak to the base (this would not be possible in array $a$ ). 


\section{When the Contracted Decision Tree Contents Cycles}

Let's see now a situation when the contracted tree, as a digraph, contents cycles. For this we have the following problem:

Officebuilding_1: Be matrix a $[1 . . n][1 . . m]$ taken as an one-storied rectangular office building. The elements of the matrix represent the offices and they store the taxes to be paid by anyone who enters the respective room. There is a door between any rooms represented by two neighbouring elements. You can enter the building only at office with position $(1,1)$ and leave it only at position $(n, m)$. Which is the minimal loss of money you can get through the building with?

For example: $n=5, m=4$.

$\begin{array}{llll}1 & 1 & 1 & 1 \\ 9 & 9 & 9 & 1 \\ 1 & 1 & 1 & 1 \\ 1 & 9 & 9 & 9 \\ 1 & 1 & 1 & 1\end{array}$

The minimal loss of money is 14 , which we got by following the shaded path.

Officebuilding_2: The same problem with the following differences:

- There are offices where they do not take money, but they give a certain amount of money ("negative tax").

- There are one-way doors (with one-side door-handles). Array b[1..n][1..m], which elements are "binary strings" with 4 characters ( ' 0 ' or ' 1 '), stores the door-codes of the offices. The first binary character of the code represents the up-door, the second the right-door, the third the down-door and the forth the left-door. For example the code "0101" means, that we can leave the office only in right and left directions.

- We assume that there is no such office-tour of the building, going along which we could increase our amount of money.

Determine the most favourable way of getting through the building.

For example: $n=5, m=4$.

Array $a$

$\begin{array}{rrrr}1 & 1 & 1 & 1 \\ 19 & 19 & 19 & 1 \\ 3 & 1 & 3 & 1 \\ -2 & 19 & 19 & 1 \\ -6 & -2 & 3 & 1\end{array}$

Array $b$

$\begin{array}{llll}0111 & 0111 & 0111 & 0011 \\ 1110 & 1111 & 1111 & 1011 \\ 1110 & 1111 & 1111 & 1011 \\ 0110 & 1111 & 1111 & 1011 \\ 0100 & 1100 & 1101 & 1101\end{array}$

The most favourable path goes through the same offices this time too, and means a loss of money of 7 (Table 1).

We are now solving a more general problem: determine the paths with the minimal cost leading from room with the coordinates $(1,1)$ to all the others. 
Table 1

Officebuilding_2 with doors and tax values

\begin{tabular}{|c|c|c|c|c|c|c|c|c|c|c|c|}
\hline \multirow{3}{*}{1} & 0 & \multirow{3}{*}{1} & \multirow{3}{*}{1} & 0 & \multirow{3}{*}{1} & \multirow{3}{*}{1} & 0 & \multirow{3}{*}{1} & \multirow{3}{*}{1} & 0 & \multirow{3}{*}{0} \\
\hline & 1 & & & 1 & & & 1 & & & 1 & \\
\hline & 1 & & & 1 & & & 1 & & & 1 & \\
\hline \multirow{3}{*}{0} & 1 & \multirow{3}{*}{1} & \multirow{3}{*}{1} & 1 & \multirow{3}{*}{1} & \multirow{3}{*}{1} & 1 & \multirow{3}{*}{1} & \multirow{3}{*}{1} & 1 & \multirow{3}{*}{0} \\
\hline & 19 & & & 19 & & & 19 & & & 1 & \\
\hline & 1 & & & 1 & & & 1 & & & 1 & \\
\hline \multirow{3}{*}{0} & 1 & \multirow{3}{*}{1} & \multirow{3}{*}{1} & 1 & \multirow{3}{*}{1} & \multirow{3}{*}{1} & 1 & \multirow{3}{*}{1} & \multirow{3}{*}{1} & 1 & \multirow{3}{*}{0} \\
\hline & 3 & & & 1 & & & 3 & & & 1 & \\
\hline & 1 & & & 1 & & & 1 & & & 1 & \\
\hline \multirow{3}{*}{0} & 0 & \multirow{3}{*}{1} & \multirow{3}{*}{1} & 1 & \multirow{3}{*}{1} & \multirow{3}{*}{1} & 1 & \multirow{3}{*}{1} & \multirow{3}{*}{1} & 1 & \multirow{3}{*}{0} \\
\hline & -2 & & & 19 & & & 19 & & & 1 & \\
\hline & 1 & & & 1 & & & 1 & & & 1 & \\
\hline \multirow{3}{*}{0} & 0 & \multirow{3}{*}{1} & \multirow{3}{*}{1} & 1 & \multirow{3}{*}{1} & & 1 & & & 1 & \\
\hline & -6 & & & -2 & & 1 & 3 & 1 & 1 & 1 & 1 \\
\hline & 0 & & & 0 & & & 0 & & & 0 & \\
\hline
\end{tabular}

As we are looking for the path with the minimal cost we can state that it must have no loops. The root of the tree structure (decision tree) which can be associated to the problem obviously represents the office with position $(1,1)$. Certain nodes will have as many sons, as the number of directions that can be taken from the respective office without making any loops. The leaves represent the end of the deadlocks. The optimal solution is represented by the path with minimal cost out of the paths leading from the root to the nodes representing the office with position $(\mathrm{n}, \mathrm{m})$. Although we cannot draw the above described decision tree due to lack of space, it is not difficult to notice that it has several identical nodes (the nodes are identified by the coordinates of the represented offices).

We overlap the identical nodes. The contracted decision tree thus obtained (see bellow), as digraph, contents cycle any more. This is due to the fact that the ancestoroffspring relationship between the nodes in the decision tree is not permanent. For example, the node $(1,2)$ is on certain branches ancestor of the node $(2,2)((1,1),(1,2),(2,2)$, $\ldots)$, on other branches the situation is opposite, node $(2,2)$ is the ancestor of $(1,2)((1,1)$, $(2,1),(2,2),(1,2), \ldots)$. Because of this, there will be a return path in the contracted decision tree between nodes $(1,2)$ and $(2,2)$.

Therefore the problem might be stated in the following way: determine the path with the minimal cost leading from node $(1,1)$ of the contracted decision tree to node $(n, m)$ (in general to all the other nodes). On the Fig. 13 we have bolded the edges of the optimal path, respectively we have shaded the corresponding offices. On the picture from the right (see Fig. 13) we have highlighted from the decision tree the subtree representing the optimal paths from the left upper corner office to all the others, applied to problem officebuilding_1.

We can consider as a general subproblem the determination of the minimal cost path leading from the left upper corner to the office with position $(i, j)$. We get the original 

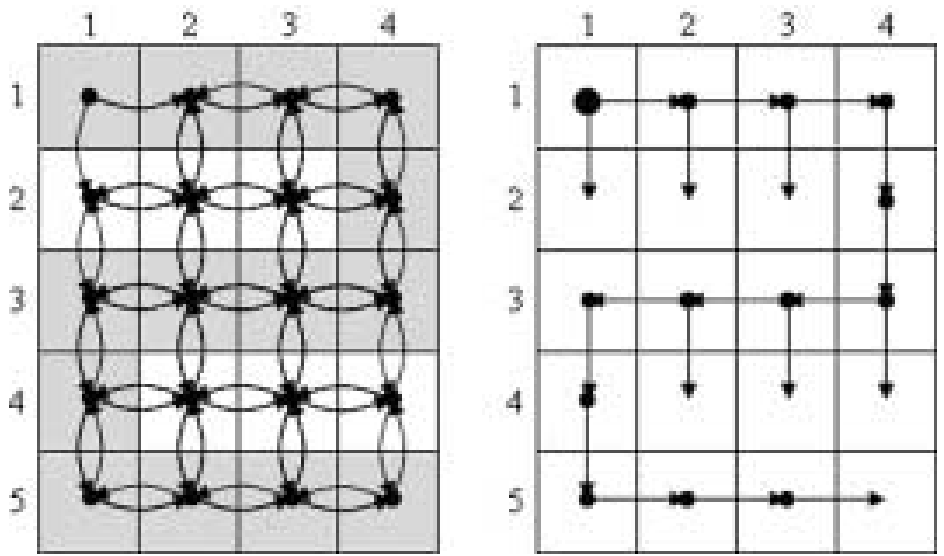

Fig. 13. The contracted decision tree attached to officebuilding problem, and the subtree representing the optimal paths.

problem for the values $i=n, j=m$, the only trivial one for values $i=1, j=1$. The cost of the optimal path leading to the office $(i, j)$ are stored in element $c[i][j]$ of the bidimensional array $c[1 . . n][1 . . n]$.

\section{Checking the Basic Principle of Optimality}

Let's assume that the minimal cost path leading to the room with coordinates $(x, y)$ is the path $\left(i_{1}=1, j_{1}=1\right),\left(i_{2}, j_{2}\right), \ldots,\left(i_{p}=x, j_{p}=y\right)$. We are going to prove that the paths with form $\left(i_{1}=1, j_{1}=1\right),\left(i_{2}, j_{2}\right), \ldots,\left(i_{k}, j_{k}\right)$ are all optimal $(k=\overline{1, p-1})$.

Let's assume that there is a path leading to room $\left(i_{k}, j_{k}\right)$ with smaller cost then the above one. Be this path $\left(u_{1}=1, v_{1}=1\right),\left(u_{2}, v_{2}\right), \ldots,\left(u_{r}=i_{k}, v_{r}=j_{k}\right)$. We can distinguish two situations. If this better path leading to room $\left(i_{k}, j_{k}\right)$ does not cross the road section $\left(i_{k+1}, j_{k+1}\right), \ldots,\left(i_{p}=x, j_{p}=y\right)$, then $\left(u_{1}=1, v_{1}=1\right),\left(u_{2}, v_{2}\right), \ldots,\left(u_{r}=\right.$ $\left.i_{k}, v_{r}=j_{k}\right),\left(i_{k+1}, j_{k+1}\right), \ldots,\left(i_{p}=x, j_{p}=y\right)$ has smaller cost than the one where we started from. But this contradicts the condition according to which we started from an optimal path. Further, if the path $\left(u_{1}=1, v_{1}=1\right),\left(u_{2}, v_{2}\right), \ldots,\left(u_{r}=i_{k}, v_{r}=j_{k}\right)$ crosses the road section $\left(i_{k+1}, j_{k+1}\right), \ldots,\left(i_{p}=x, j_{p}=y\right)$, let's say in room $\left(u_{f}=i_{g}, v_{f}=j_{g}\right)$ $(1<f<r, k<g<p)$, then path $\left(u_{1}=1, v_{1}=1\right),\left(u_{2}, v_{2}\right), \ldots,\left(u_{f}=i_{g}, v_{f}=\right.$ $\left.j_{g}\right),\left(i_{g+1}, j_{g+1}\right), \ldots,\left(i_{p}=x, j_{p}=y\right)$ will have smaller cost than the original path, which is again a contradiction. So, the optimal paths are built from optimal subpaths.

\section{Establishing the Optimal Solution in the 1. Office Building}

Here is the recursive formula which describes the structure of the optimal solution:

$$
c[1][1]=a[1][1]
$$


otherwise

$$
\begin{aligned}
c[i][j]= & a[i][j] \\
& +\min (c[i-1][j], c[i][j+1], c[i+1][j], c[i][j-1])
\end{aligned}
$$

assuming that the rooms with the respective positions exist

As we can see, we haven't charged the general formula with conditions refering to parameters $i$ and $j$. It is well perceptible from the pictures from which directions can we enter into certain rooms.

In accordance to the basic principle of optimality, the minimal cost path leading to office $(i, j)$ can only go through offices where the "best" path has smaller cost than to office $(i, j)$. This means that in order to determine the array-element $c[i][j]$ we effectively need those array-elements, which represent smaller value minimal cost paths. All this refer to the fact that the elements of array $c$ have to be filled in the increasing order of the optimal values of the optimal paths leading to the represented offices

In case of the first office building this can be ensured with a data structure called priority-queue. The elements of the queue will be the elements of array $c$ (we remember that these, as well as the corresponding nodes of the contracted decision tree, represent the subproblem of the determination of the minimal cost path leading to the respective offices) in the increasing order of the values stored in them.

This is shown by the Fig. 14. With black we have marked those array-elements which have already been removed from the queue. These represent the offices where the value of the minimal cost path leading to them has already been determined. In the queue there are those offices which can be reached from the "black offices". We marked them with
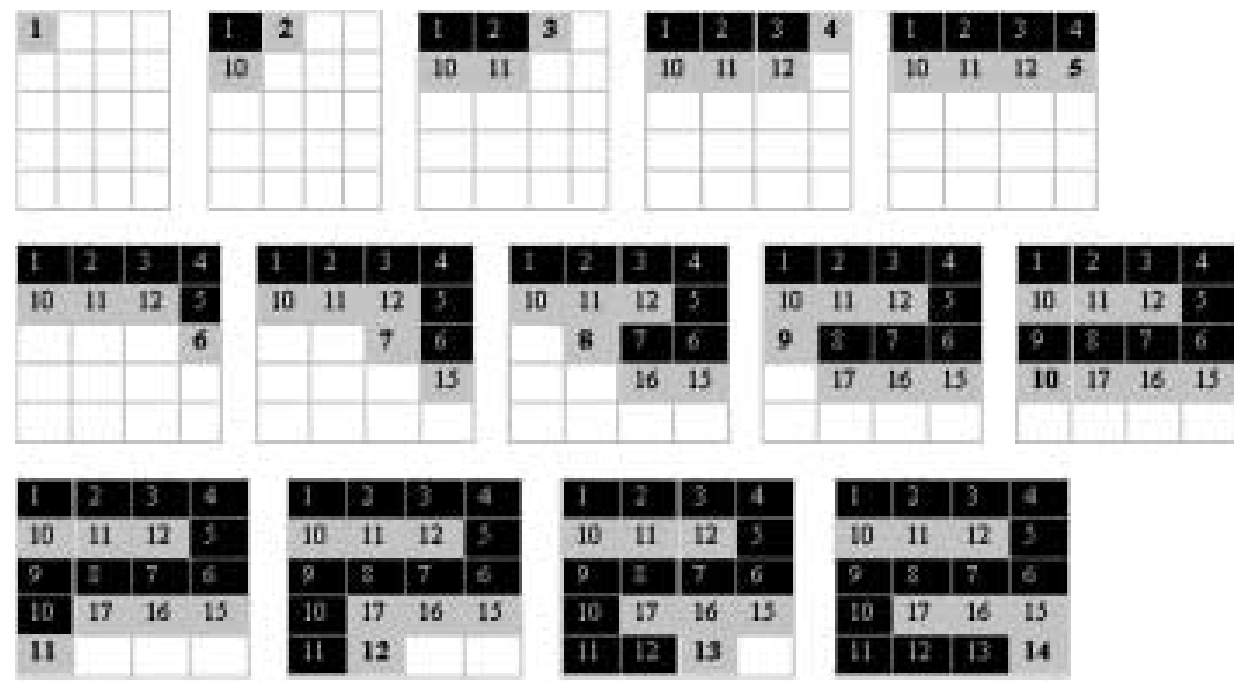

Fig. 14. The filling up of the array $c$ attached to officebuilding problem. Black elements - which have been removed from queue. Gray elements - which are in queue. White elements - which monetary can't be reached from the black elements. The highlighted element - the first element in queue. 
grey. The elements corresponding to the "grey offices" store the minimal costs we can get to them with, crossing exclusively black rooms. We have highlighted the highest priority element of the queue, the "first in the queue". We have left white those offices which cannot be reached through the black offices.

At the beginning the queue-structure contains the office with position $(1,1)$. This is reflected in the array by making element $c[1][1]$ grey and it is filled with the value $a[1][1]$ (this is the tax claimed in this office). Following this, the algorithm executes at each step the following operations on the office which is first in the queue (should its coordinates be $\left.\left(i_{-} e, j_{-} e\right)\right)$ :

- It changes the colour of the office which is the first in the queue into black, (thus it is erased from the queue, as one to which the minimal cost path has been found). It is obvious that the value $c\left[i_{-} e\right]\left[j_{-} e\right]$ represents a minimal cost path from the fact that all the other offices (the grey ones and the white ones) either cannot be reached through the offices which are already black (the white ones) or they can only be reached on more expensive paths (the other grey ones).

- Should the office which is the first in the queue have such a grey neighbour to which there is a path through it with a lower cost than the one crossing the offices being black until now, then we refresh the corresponding array-element (thus the respective office obviously advances in the priority list). If we mark the position of the respective grey neighbour with $\left(i_{-} s z, j \_s z\right)$ then the operation is the following:

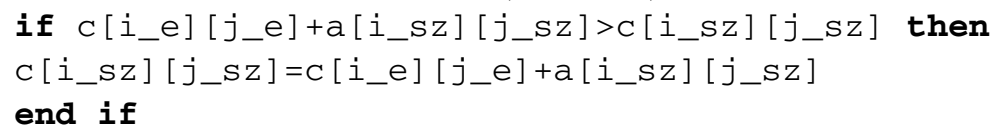

In the case of this problem specially this situation cannot occur.

- We enter the "white offices" reachable from the office which is the first in the queue in the priority list (we change the colour of the correspondent array-element into grey and we fill it up). Should the coordinates of such a white neighbour be $\left(i_{-} f, j_{-} f\right)$, then $c\left[i_{-} f\right]\left[j_{-} f\right]=c\left[i_{-} e\right]\left[j_{-} e\right]+a\left[i_{-} f\right]\left[j_{-} f\right]$.

We repeat this until the office with position $(n, m)$ becomes first in the queue or, in case we want the shortest path to every office, until the queue becomes empty.

It can be proved that the algorithm presented above will determine the minimal cost path leading to certain offices really in the order of the optimal values.

\section{Establishing the Optimal Solution in the 2. Office Building}

It is not difficult to realize that in this case the above presented algorithm would not lead to the optimal solution. Due to the presence of the "negative taxes" there can be a path to the grey office being for the moment first in the queue, with lower cost -involving "negative tax" white offices - than the minimal cost path touching exclusively black offices.

What order should we fill up the elements of array $c$ in this case? As there is no possibility of determining a correct order of calculating the minimal cost paths neither in advance (there is no topological order) nor "during the run" (with the help of a priority list), we are using a different method. 
For a first go we fill up array $c$ with the values given by traversing it row by row (from top to bottom, left to right). Of course we can take into consideration only neighbours $c[i-1][j]$ és $c[i][j-1]$ when filling element $c[i][j]$ during the traversing (only these have already been filled up), although on the optimal paths there are sections pointing to the left, respectively upwards (short: backwards-edges), then elements $c[i][j+1]$ és $c[i+1][j]$ should essentially be taken into consideration. For example in the above presented problem calculating the value $c[3][3]$ assumes to know the value of $c[3][4]$. Furthermore we can also say that after this filling up we get the shortest path only to those rooms, in case of which they contain edges only pointing forwards (to the right, respectively down). What is the solution? We go again and again through array $c$, refining it. During these further traversings we overwrite those array-elements to which there is a more advantageous path from a neighbouring element. This time we can take into consideration all four directions. When a new traversing does not bring any alterations, it means that we have reached the optimal solution. We can notice that there are as many refining traversings as the number of backwards-edges in the subtree of the optimal paths. If we can still refine after $\mathrm{nm}$ (number of the nodes of the contracted decision tree) traversings, it means that in contradiction to the conditions of the problem, there is such an office-tour going round which our money is becoming more

In what follows we are presenting the content of array $c$ after the filling, respectively refining traversings. We have written in bold those elements which already represent optimal paths. In the attached pictures we wanted to show the way the subtree representing the optimal solution is being built, in accordance to the principle of optimality from the bottom toward the top, from the simple toward the complex. In bold we have represented the part of the tree which has already been built until the given step.

The content of array $a$ in the problem:

$\begin{array}{rrrr}1 & 1 & 1 & 1 \\ 19 & 19 & 19 & 1 \\ 3 & 1 & 3 & 1 \\ -2 & 19 & 19 & 1 \\ -6 & -2 & 3 & 1\end{array}$

The content of array $c$ after the filling traversing (see Fig. 15):

$\begin{array}{rrrr}\mathbf{1} & \mathbf{2} & \mathbf{3} & \mathbf{4} \\ \mathbf{2 0} & \mathbf{2 1} & \mathbf{2 2} & \mathbf{5} \\ 23 & 22 & 25 & \mathbf{6} \\ 21 & 40 & 44 & \mathbf{7} \\ 15 & 13 & 16 & 8\end{array}$

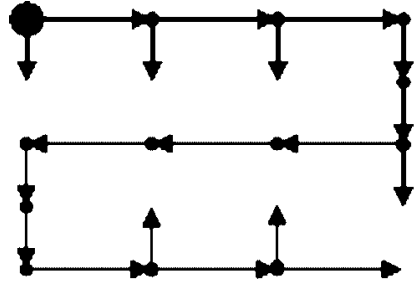

Fig. 15. After the filling traversing. 
The content of array $c$ after the first refining traversing (see Fig. 16):

$\begin{array}{rrrr}\mathbf{1} & \mathbf{2} & \mathbf{3} & \mathbf{4} \\ \mathbf{2 0} & \mathbf{2 1} & \mathbf{2 2} & \mathbf{5} \\ 23 & 22 & \mathbf{9} & \mathbf{6} \\ 21 & 32 & 26 & \mathbf{7} \\ 15 & 13 & 11 & 8\end{array}$

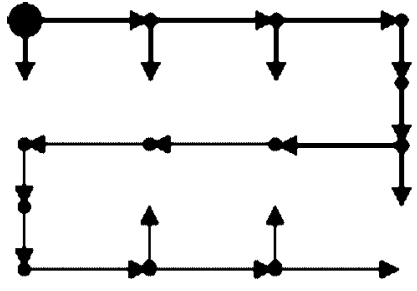

Fig. 16. After the first refining traversing.

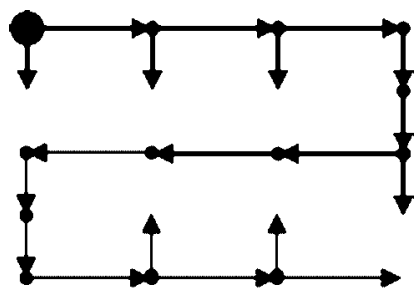

Fig. 17. After the second refining traversing.

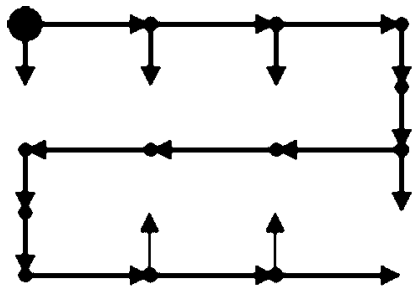

Fig. 18. After the third refining traversing.

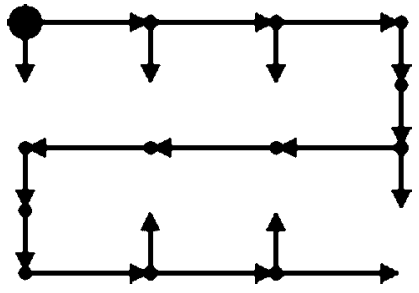

Fig. 19. After the final refining traversing.

Array $c$ in its final form (after its fifth traversing) (see Fig. 19):

\begin{tabular}{rrrr|}
$\mathbf{1}$ & $\mathbf{2}$ & $\mathbf{3}$ & $\mathbf{4}$ \\
$\mathbf{2 0}$ & $\mathbf{2 1}$ & $\mathbf{2 2}$ & 5 \\
$\mathbf{1 3}$ & $\mathbf{1 0}$ & $\mathbf{9}$ & $\mathbf{6}$ \\
$\mathbf{1 1}$ & $\mathbf{2 2}$ & $\mathbf{2 5}$ & $\mathbf{7}$ \\
$\mathbf{5}$ & $\mathbf{3}$ & $\mathbf{6}$ & $\mathbf{7}$
\end{tabular}

\section{"Optimal Division - Optimal Conquest"}

So far we have been using dynamic programming in the case of problems where with each step it was reduced to a similar, simpler problem. With the term "reduced" we wanted to express the fact that by each decision we solve a certain part of the problem. Therefore we can say that at each level the problem contains a solved and an unsolved part. These were the prefix respectively suffix subproblems belonging to a certain state. We could 
say that as a result of the sequence of decisions $D_{1}, D_{2}, \ldots, D_{n}$ the problem was solved step by step. The question only was how to make these decisions, in order to get the optimal solution. At a first glance these problems seem to be "greedy problems", but as the basic principle of the greedy selection is not applicable to them, "we have to" apply the dynamic programming.

An other field of the dynamic programming consists of problems which are closer to the "divide and conquer" technique. The essence of the "divide and conquer" is that it divides the problem into two or more similar, simpler subproblems and builds up the original problem's solution from their solutions (of course the procedure is the same for the subproblems, until we get trivial subproblems). What is the situation if the division of the problem into subproblems can be achieved in several ways? If this is the case, the question of the optimal division arises! In such situation the point is more than a simple "divide and conquer" problem. If at each step the optimal "cut" could be made with a greedy decision, then we can say that the greedy technique facilitated the work of the "divide and conquer". Should we not have enough information for the greedy cuts, but the principle of optimality is valid for the task of dividing the problem, dynamic programming might help the "divide and conquer" technique to find the optimal solution.

So we are speaking about a strategy which builds dynamic programming into the "divide" stage of the "divide and conquer" technique (this is called mixed method by Tudor Sorin, 1997). This means that the optimal sequence of decisions $D_{1}, D_{2}, \ldots, D_{n}$ characteristic for optimization problems basically means the optimally breaking down of the problem into subproblems. By each decision the problem breaks down into similar, simpler subproblems ( in contradiction with the situation when it was reduced to one similar, simpler problem). When we say optimally division into subproblems, we consider that it is optimal because it involves the building of the optimal solution in the "conquer" stage of the "divide and conquer". As the sequence of decisions $D_{1}, D_{2}, \ldots, D_{n}$ only means the way the problem optimally breaks into subproblems, in an intermediate Si stage (that we reached after a sequence of $D_{1}, D_{2}, \ldots, D_{i}$ decisions) we cannot speak about a part of the problem which has already been solved (prefix subproblem). Therefore, in such case only the leaves-root oriented version of the dynamic programming can be taken into consideration. This is in accordance with the fact that the "divide and conquer" technique solves a father-subproblem only after having solved the son-subproblems (according to the above introduced terminology by subproblems we mean suffix type subproblems).

\section{The Principle of Optimality on a II Type Decision Tree}

Let's assume again that $D_{1}, D_{2}, \ldots, D_{n}$ is the sequence of decisions which optimally breaks the problem up into subproblems. For simplicity we presume that the current subproblem falls into two further subproblems apart by each step ( until we get trivial subproblems). How does the basic principle of optimality become evident in case of such a problem? If we assume that after decision $D_{1}$ the problem breaks up into two subproblems so that their further division is ensured by the subsequence of decisions $D_{2}, \ldots, D_{k}$ 
respectively $D_{k+1}, \ldots, D_{n}$, each of these should also be optimal (in the sense that they break up the corresponding subproblems in a way which leads to the optimal solution).

We could say that in so far as the sequence of decisions leading to the optimal solution is $D_{1}, D_{2}, \ldots, D_{n}$, then the subsequence of decisions $D_{2}, \ldots, D_{k}$ and $D_{k+1}$ for any $k(k=\overline{2, n-1})$ is also optimal. Continuing this idea, it assumes that the couples of subsequence of decisions $D_{3}, \ldots, D_{k 1}$ and $D_{k 1+1}, \ldots, D_{k}$, as well as $D_{k+2}, \ldots, D_{k 2}$ and $D_{k 2+1}, \ldots, D_{n}$ for a certain $k_{1}\left(k_{1}=\overline{3, k-1}\right)$ respectively $k_{2}\left(k_{2}=\overline{k+2, n-1}\right)$ are also optimal. And so on ....

It is not difficult to realize that in so far as this situation occurs, the optimal solution of the general subproblem is represented by a section of sequence of decisions $D_{i}, D_{i+1}, \ldots, D_{j}$. What does it mean in this situation to build from down upwards? We solve the subproblems in the increasing order of the length of the sections of sequence of decisions representing their solution.

This situation is different from the one where by each decision the problem was reduced to only one similar simpler subproblem and the validity of the basic principle of optimality was obvious. It might occur that the optimization of the subproblems we have broken the problem up to, get into conflict.

\section{Dynamic Programming on the II Type Decision Tree}

We make this version of the dynamic programming easier to grasp through a solved problem.

Mirrorword: A string is given. Divide it into a minimal number of mirrorwords.

EXAMPLE. Be the string: $a b a b b$. We can see that it can be divided into mirrorwords in several ways:

(a) (b) (a) (b) (b), (a) (b) (a) (bb), (aba) (bb), (a) (bab) (b), (aba) (b) (b)

The optimal solution is obviously represented by the third version.

The basic idea is that if the string is not a mirrorword itself, we cut it into two, thus bringing the problem back to the division into mirrorwords of two shorter strings. Having done this, the number of minimal mirrorwords of the original problem is given by the optimal sum of the two parts. This sound like a "divide and conquer" algorithm. But it is not only that, because the cuts can be performed in general in several ways and there is no possibility to decide (with a greedy decision) which is the one leading to the optimal solution. In the followings we are going to show the decision tree associated to the problem, applied to the example. The nodes of the tree contain the starting and ending indexes of the corresponding substring within the string. For example the node (1-5) represents the whole string ( $a b a b b)$, and (3-5) represents the substring $a b b$.

The decision tree (see Fig. 20) differs from the usual because it presents the way the problem can be broken up into its subproblems. For example the original problem can be cut into two in four different ways: a|babb, ab|abb, aba|bb, abab|b. The optimal cut is the third one, (which in this case also represents the solution), the one that leads to the 


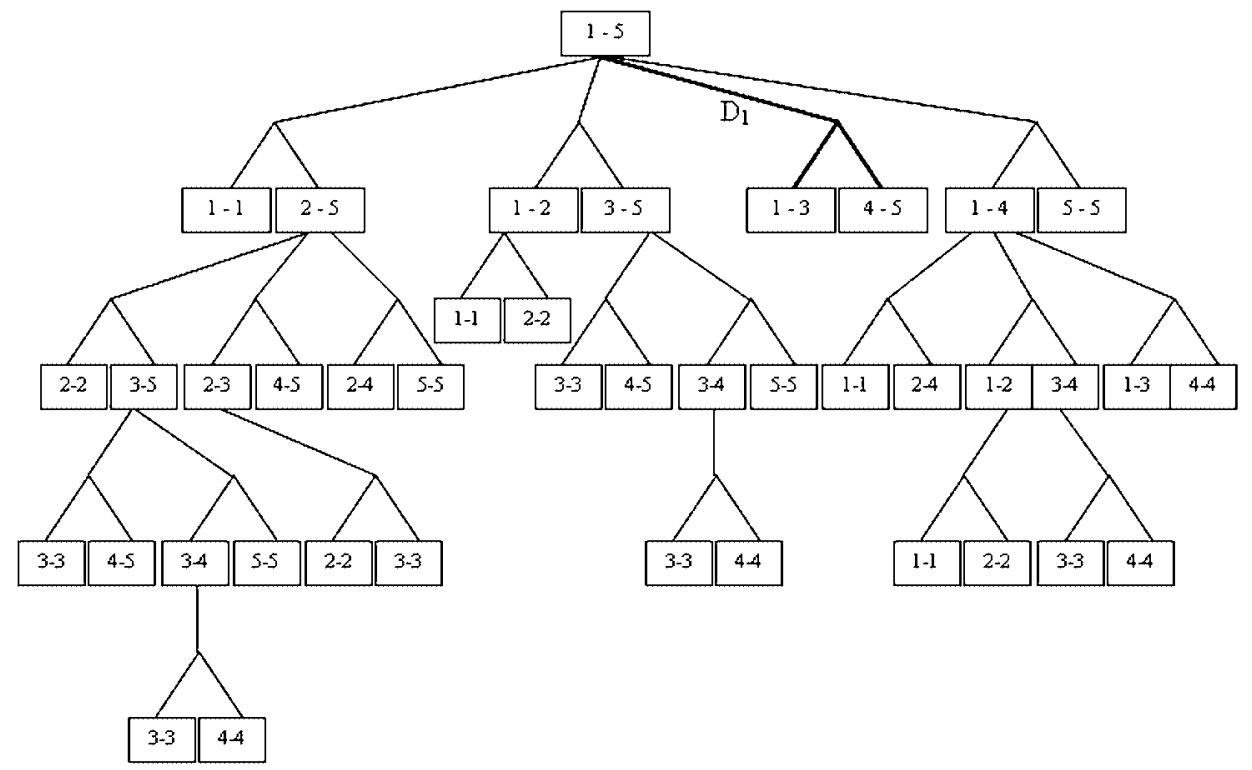

Fig. 20. The decision tree associated to mirrorword problem.

substrings (1-3) and (4-5), as these are already mirrorwords. We can notice that all the leaves of the tree - and only them - represent mirrorwords.

We can also read from the tree that the general form of the subproblems is: the optimal division of the substring $(i-j)$ into mirrorwords. As in the general form there are two independent parameters and $i \leqslant j$, we are going to use the elements from the main diagonal and above the main diagonal of a bidimensional array $c[1 . . n][1 . . n](n-$ the number of the characters of the string) to store the optimal values of the subproblems. The array-element $c[i][j]$ will store the minimal number of mirrorwords corresponding to the optimal division of segment $(i-j)$.

In accordance to what has been said before we can notice that if we overlap the identical nodes of the decision tree, the nodes of the contacted decision tree thus obtained can be rearranged so that they form a shape corresponding to a triangle above the main diagonal of a $n \times n$ sized bidimensional array (the number of the nodes of the decision tree which differ from each other is $n(n+1) / 2)$. This is noticeable in Fig. 21. The root of the tree gets into the $n$th position of the 1st row, in the same way as the optimal value of the original problem will be stored in array $c[1][n]$. The strings containing one element are obviously mirrorwords, therefore the elements of the main diagonal store in any case the optimal values of trivial subproblems, but of course there can be other array-elements the subproblems corresponding to them also being trivial in case the respective substring is a mirrorword itself. Every mirrorword-substring is represented by leaves in the decision tree and in the contracted tree as well. 


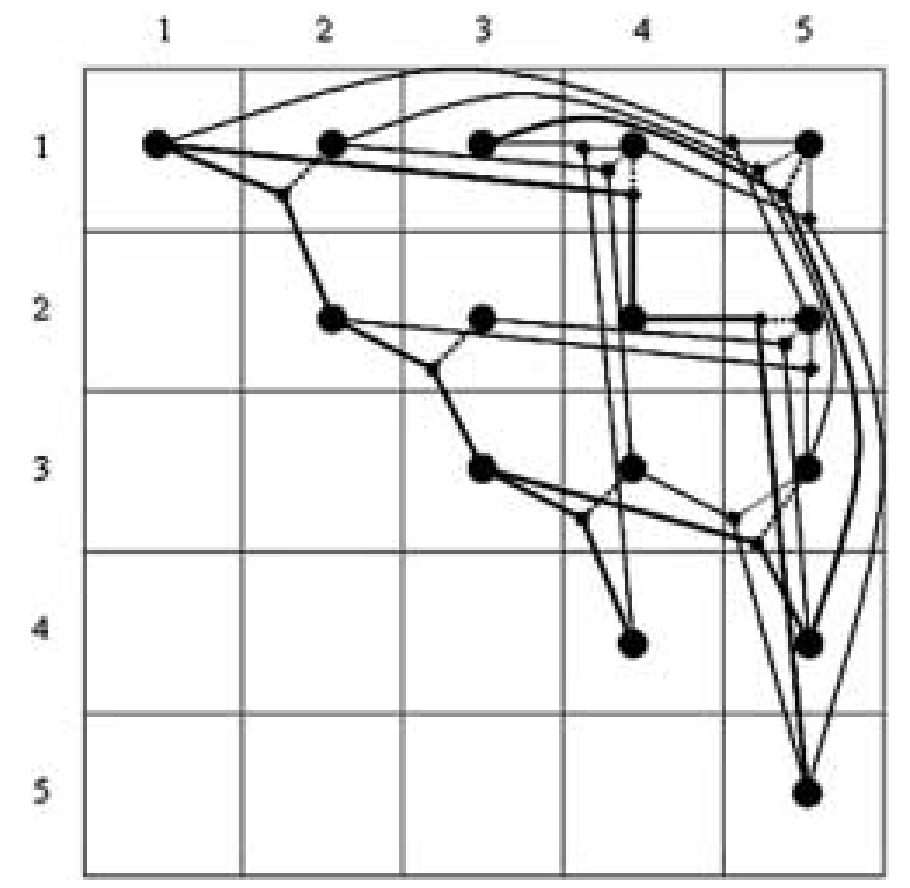

Fig. 21. The contracted decision tree attached to mirrorword problem.

\section{The Check of the Basic Principle of Optimality}

As the two subproblems created after a certain cut are independent from each other, and thus their optimal solution cannot get in conflict, the basic principle of optimality obviously applies here. Namely: If the segment $(i-j)$ is divided after the first optimal division into the pair $(i-k)$ and $(k+1, j)$ then their further cutting through the optimal division is certainly optimal concerning them, too. Since had one of them a better division than the one through which it is divided by the optimal division of the segment $(i, j)$, then choosing this one we would get to a better division for segment $(i, j)$, too. But this contradicts the condition that we started from the optimal division of segment $(i-j)$. Therefore the division of the original string can be determined from the optimal division of the substrings.

\section{The Principle of Optimality in the "Contracted Decision Tree"}

Heading from the leaves to the root we leave on each fathernode that couple of sonnodes, whose corresponding couple of subproblems have the minimal total value of optimums (we prune the rest from the tree). We have represented the optimal choices on the contracted tree in bold. 


\section{The Principle of Optimality in the Array Storing the Optimal Values}

We fill the elements from the main diagonal, respectively from above the main diagonal of array $c$ according to the following recursive formula:

$$
c[i][j]=\left\{\begin{array}{lc}
1, & \begin{array}{l}
\text { if the string-segment }(i-j) \\
\text { is a mirrorword, }
\end{array} \\
\min _{i \leqslant k<j}\{c[i][k]+c[k+1][j]\}, & \text { in the opposite case. }
\end{array}\right.
$$

As this formula is suggesting, the elements should be filled up in such an order, that when the filling up of element $c[i][j]$ is to follow, the couples of elements $c[i][k]$ and $c[k+1][j]$ $(i \leqslant k<j$ ) should be already filled up. Such an order is possible if starting from the elements next to the main diagonal, we advance from diagonal to diagonal until element $c[1][n]$.

If we would like to get also the optimal division into mirrorwords (not only the minimal number of mirrorwords), array $c$ contains enough information for us to divide our string into mirrorwords by greedy cuts as a problem of "divide and conquer" starting from the root. It is recommendable, when we fill up array $c$, to store the optimal $k$-value belonging to the optimal division of segment $(i-j)$ into array-element $c[j][i]$ (anyhow, we have not used the triangle under the main diagonal yet), and thus we don't have to choose again the optimal cuts with the aid of a min function. If we do this, the string-segment $(i-j)$ is divided optimally into the couple of strings $(i, c[j][i])$ and $(c[j][i]+1, j)$ at the reconstruction of the solution. If in case of $i<j$ the segment $(i-j)$ is a mirrorword itself, this should be shown by the value 0 in element $c[j][i]$.

In this case the optimal solution in the decision tree is not represented by one of the root-leaf paths, but by the binary (because by each cut we get two subproblems) subtree corresponding to the optimal division. We can see this in Fig. 22. highlighted from the decision tree (for the example problem the binary tree of the optimal solution only consists of root and first level sons), and we have drawn this in array $c$ too, which - as we have already discussed - contains on the main diagonal and above the main diagonal the optimal values of the subproblems and under the main diagonal the optimal $k$-values.

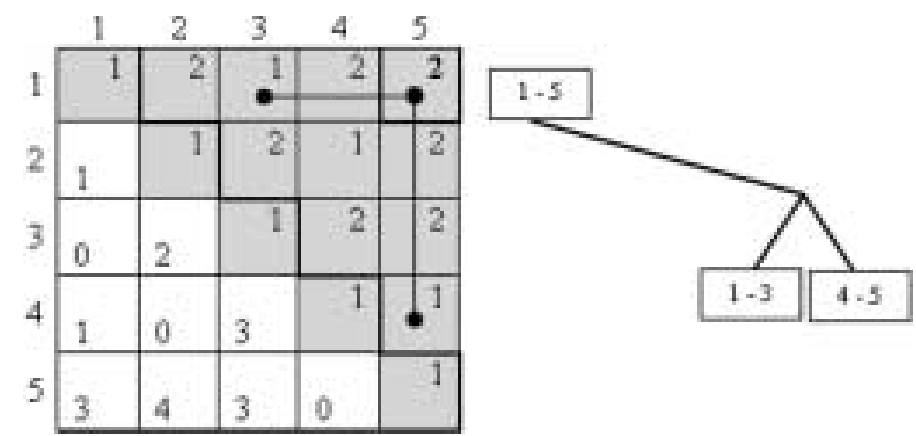

Fig. 22. The filled up array $c$ attached to mirrorword problem. The subtree which represents the optimal solution. 


\section{Conclusions}

The strength of the above presented approach is its illustrability. This is extremely important in teaching dynamic programming. But this is something more than a didactic point of view. This illustrative approach can be very useful in understanding the dynamic programming as a method and in the classification of its different versions (I and II type decision trees).

The terminology used is also very illustrative: contracted decision tree, prefix and suffix subproblems, root-leaves, respectively leaves-root oriented dynamic programming, "optimal division - optimal conquest".

Of course there is no question of having completely exhausted the analysis of the topic of dynamic programming from this point of view. We meant this paper to be an introduction, leaving space for more studies.

\section{References}

Andone, R., and I. Garbacea (1995). Fundamental Algorithms a C++ Perspective. Libris Press, Cluj-Napoca (in Romanian).

Cormen, T.H., C.E. Leirserson and R.L. Rives (1990). Introduction to Algorithms. The Massachusetts Institute of Technology.

Kátai, Z. (2005). "Upperview" algorithm design in teaching computer science in high schools. In Teaching Mathematics and Computer Science. University of Debrecen, Hungary, pp. 222-240.

Tudor, S. (1997). Programming Techniques. L\&S InfoMat Press, Bucuresti (in Romanian).

Z. Katai is an assistant professor at Sapientia-Hungarian University of Transylvania. $\mathrm{He}$ is going to obtain the PhD degree on February 15, 2007. His reasearch interests are teaching methods (especially in informatics), dynamic programming, the role of senses in teaching-learning process.

\section{Dinaminio programavimo strategijos, sprendžiant optimizavimo uždavinius, remiantis medžio algoritmu}

\section{Zoltan KATAI}

Straipsnio tikslas - pristatyti patikrintas dinaminio programavimo strategiju charakteristikas, remiantis medžio algoritmu ir pasiūlyti aiškią priemonę tụ strategijų analizei ir klasifikacijai, padedančiai perprasti šios programavimo technologijos esmę. 